\title{
Breathability Enhancement of Electrospun Microfibrous Polyurethane Membranes through Pore Size Control for Outdoor Sportswear Fabric
}

\author{
Seong Kyung Hong ${ }^{1}$, Geunbae Lim $^{1, *}$ and Seong J. Cho ${ }^{1,2, * *}$ \\ 'Department of Mechanical Engineering, Pohang University of Science and Technology \\ (POSTECH), Pohang 790-784, Republic of Korea \\ ${ }^{2}$ Polymer Research Institute, Pohang University of Science and Technology (POSTECH), \\ Pohang 790-784, Republic of Korea
}

(Received July 17, 2014; accepted December 8, 2014)

Key words: electrospinning, polyurethane, microfibrous membrane, breathable fabric, pore size control

Electrospinning of polyurethane (PU) is widely used to fabricate breathable fabrics for applications in outdoor sportswear, and can be used to produce a highly porous structure, which is an essential property of a breathable fabric. To increase the breathability of the fabric, herein we describe the use of a metal mesh as the ground electrode in place of the conventional planar electrode during electrospinning. This electrode geometry results in an electric field that leads to the electrospun fibers being predominantly stacked over the metal wires of the mesh, with fewer fibers over the holes, resulting in larger pores in the membrane. The average pore size and thickness of the membrane were compared with those of a membrane fabricated using a conventional planar electrode. A quantitative analysis performed according to the Korean Industrial Standards (KS) indicated improved breathability.

\section{Introduction}

The fabrication of fabrics for use in various functional applications has recently become an area of interest in the clothing and sportswear industry. ${ }^{(1,2)}$ The combination of breathability and water repellency, i.e., being able to release sweat from beneath the fabric while remaining waterproof to rain, is a desirable property.

Fabricating a fabric with a hydrophobic surface, so that it is water-repellent, has been demonstrated using various methods, including exploiting naturally hydrophobic polymers such as polyurethane (PU), ${ }^{(1,2)}$ or hydrophobic coatings, such as

*Corresponding author: e-mail: limmems@postech.ac.kr

${ }^{* *}$ Corresponding author: e-mail: lelax@postech.ac.kr 
polytetrafluoroethylene (PTFE). ${ }^{(3)}$ Breathability is one of the most important properties for applications in outdoor sportswear. To achieve breathability, a microporous structure is necessary, as this allows passage of vapor-phase molecules. Such a microporous structure can be achieved using a facile fabrication method known as electrospinning.

Electrospinning is a simple and versatile method that can be used to produce ultrafine continuous fibers by exploiting electrohydrodynamic forces. This method has been widely used to fabricate homogeneous membranelike nonwoven webs, which can be used as fabrics. ${ }^{(4-7)}$ A high voltage is applied to a polymer solution that is typically stored in a syringe. During the process, charge accumulates on the polymer solution, eventually erupting in a jet towards the grounded electrode from the apex of a conical meniscus, commonly known as a Taylor cone. ${ }^{(8)}$ Although the jets in a Taylor cone are stable near the tip of the nozzle, the liquid electrospun jets typically experience instability, which leads to solidification of the jet and thinning of the fibers as the jet approaches the collector. ${ }^{(9)}$ Because of this instability, as the electrospun fibers are stacked on the grounded electrode, they inevitably form a randomly oriented fibrous structure, which is porous. ${ }^{(10-13)}$ Although the electrospinning process itself is capable of fabricating a highly porous breathable membrane, there is scope for increasing the breathability of the membrane if the random orientation of the stacked fibers can be controlled to form larger pores.

There have been a number of attempts to control the random stacking of the fibers, and much interest in controlling the orientation or patterning of micrometer- or nanometer-scale fibers. Most approaches have either varied the applied electric field ${ }^{(14,15)}$ or the geometry of the collector electrode. ${ }^{(16-24)}$ Controlling the applied electric field appears to be a promising means of controlling the pattern of the electrospun membranes. The chaotic and rapid motion of the jet cannot be suppressed at high voltages. Recently, Chang et al. developed a continuous near-field electrospinning technique with an extremely low voltage and a short nozzle-collector separation. ${ }^{(15)}$ Bending instabilities were suppressed, allowing improved control over the deposition of the electrospun fibers using an $x-y$ translation stage.

The structure of the electrospun membranes has also been controlled by varying the geometry of the collector. Li et al. used multiple electrodes interspersed with nonconductive gaps, and found that the fibers naturally aligned across the gaps. ${ }^{(18)}$ More recently, there have been reports of the effects of the void-gap geometry, including round, square, triangle, and rectangular holes. In particular, Vaquette and CooperWhite used a metal mesh collector with square and round holes of various sizes. The fiber diameter, pore size, and mechanical properties of the electrospun membranes were investigated as a function of the geometry of the collector. ${ }^{(24)}$

We reported previously the fabrication of breathable fabrics using a superhydrophobic stretchable fibrous membrane. ${ }^{(25)}$ The superhydrophobicity of the membrane resulted in water repellency significantly greater than that of conventional functional fabrics, such as Gore-Tex ${ }^{\circledR}$. Furthermore, the improved stretchability allowed the membrane to be deformed, exhibiting mechanical strain of up to $300 \%$. These membranes have scope for superior performance for applications in outdoor sportswear compared with conventional fabrics because of the improved water repellency and stretchability. 
There also have been previous demonstrations of the use of patterned electrodes to fabricate desired shapes via electrospinning. Cho et al. demonstrated that electrospun nanofibers can be deposited selectively with microscale resolution, and can be aligned onto flexible insulators, by controlling the geometry of the electrode. ${ }^{(23)}$ These reports suggest considerable scope for improving the breathability of waterproof fabrics by controlling the structure of electrospun membranes.

In this work, we integrate a collector electrode with a mesh geometry with empty square holes for fabrication of PU membranes with large pores to achieve improved breathability for applications in outdoor sportswear. Typical electrospinning processes employ a planar ground electrode (commonly aluminum foil) as the collector. The constant electric field on this surface leads to a random orientation of the electrospun fibers. Because of this random orientation, porous membranes can be produced. Here, we show that improved breathability can be achieved using a metal mesh as the ground electrode. The electrospun fibers are attracted toward the metal of the mesh, leading to a structured membrane with fewer fibers over the holes and more fibers over the metal wires. This leads to larger pores (i.e., larger areas where there are relatively few fibers), which leads to the improved breathability of the membrane. The regions of the membrane where there are few fibers not only transmit more gas molecules but also visible light, making the membrane partially transparent. Furthermore, the thick portions of the membrane (over the metal wires in the mesh) enable it to be structurally reinforced, making the membrane particularly resilient to mechanical strain.

\section{Materials and Methods}

\subsection{Electrospinning of $P U$}

PU (Pellethane 2363-80AE, Lubrizol, USA) was chosen as the main fiber for electrospinning owing to its elasticity. To prepare the solution for electrospinning, 13$\mathrm{wt} \%$ PU was dissolved in tetrahydrofuran (THF) and dimethylformamide (DMF), which were mixed at a volume ratio of 60:40. The PU was allowed to dissolve for at least $12 \mathrm{~h}$.

The electrospinning setup is illustrated in Fig. 1(a). Using a high-voltage source, a potential of $9.5 \mathrm{kV}$ was applied between the polymer solution at the tip of the syringe
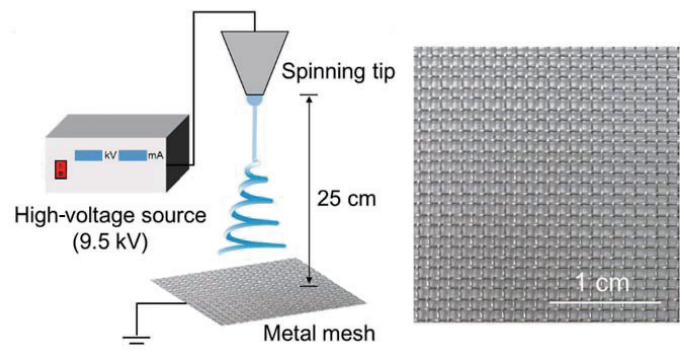

Fig. 1. (Color online) (a) Schematic diagram of the electrospinning setup utilizing a metal mesh as the collector. (b) Optical image of the actual metal mesh used in the electrospinning process. 
and the ground electrode, which was formed from a metal mesh. A distance of 25 $\mathrm{cm}$ separated the syringe tip and the metal mesh collector. As shown in Fig. 1(b), the metal mesh was composed of an array of interwoven metal wires and empty holes. The wires had a diameter of $400 \mu \mathrm{m}$, and the dimensions of the holes were $1 \times 1 \mathrm{~mm}^{2}$. The electrospinning process described above was carried out using a planar sheet of aluminum foil as the ground electrode for collecting electrospun PU as a control. The electrospun PU membranes were detached from the collector and cut into $10 \times 10 \mathrm{~cm}^{2}$ pieces for assessment of breathability.

\subsection{Membrane pore size}

The pore size of the membranes was measured using scanning electron microscopy (SEM) images taken from five areas of each membrane, with a magnification of $1000 \times$. The 10 foremost fibers were selected manually, and considered to form a single layer. Figure 2(a) shows the 10 foremost fibers selected as a single layer of an electrospun membrane on the aluminum foil collector. This image was processed using ImageJ software to calculate the average pore size, as shown in Fig. 2(b). Figures 2(c) and 2(d) show the pore size assessment of a membrane electrospun on the metal mesh collector. To directly compare the pore sizes of both membranes, the average pore areas were used to calculate the average pore diameter, assuming that all pores were circular.

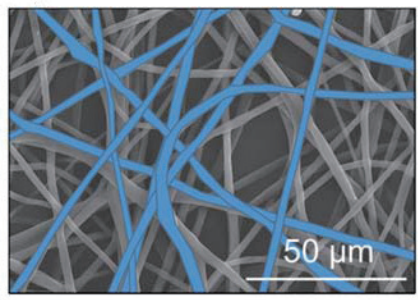

(a)

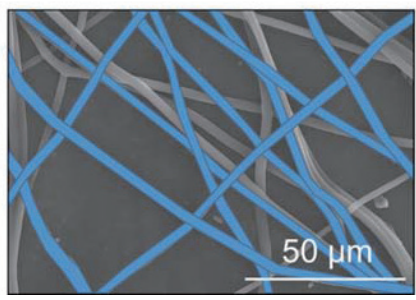

(c)

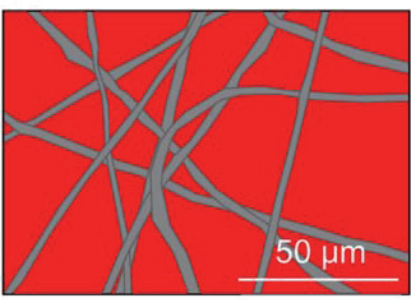

(b)

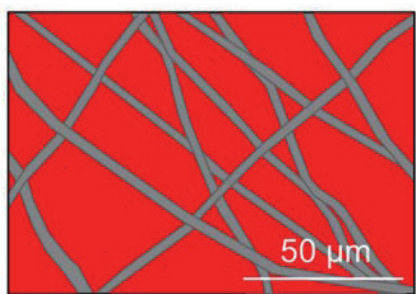

(d)

Fig. 2. (Color online) (a) SEM image of PU-foil membrane with 10 foremost fibers selected as blue trails. The selected fibers were considered as a single layer. (b) Imaged processed single layer from (a) that shows its pores as red areas. (c) SEM image of PU-mesh membrane with 10 foremost fibers selected as blue trails. The selected fibers were considered as a single layer. (d) Imaged processed single layer from (c) that shows its pores as red areas. 


\subsection{Breathability}

The electrospun PU membranes were tested for breathability via a quantitative analysis according to the Korean Industrial Standards (KS) of the Korean counterpart of International Organization for Standardization (ISO) for fabrics. The test used to evaluate breathability is termed the "the water-cup method". Figure 3 shows a schematic diagram of the test. The electrospun PU membrane covered the cup, which had an 8-cmdiameter opening and contained water at $40{ }^{\circ} \mathrm{C}$. The ambient air above the membrane was at $40{ }^{\circ} \mathrm{C}$ and $50 \%$ relative humidity. The test quantifies the water vapor that passes through the membrane in $1 \mathrm{~d}$. This is known as the moisture vapor transmission rate (MVTR); a higher MVTR corresponds to superior breathability.

\section{Results and Discussion}

Two types of membrane were prepared: a PU membrane formed using the metal mesh (PU-mesh) and a PU membrane formed using the planar aluminum foil collector (PU-foil).

\subsection{Pore size}

Figure 4 shows optical images of both membranes, together with SEM images of specific areas of each membrane. An optical image of the PU-mesh membrane is shown in Fig. 4(a). The membrane was composed of thick regions, which clearly resemble the
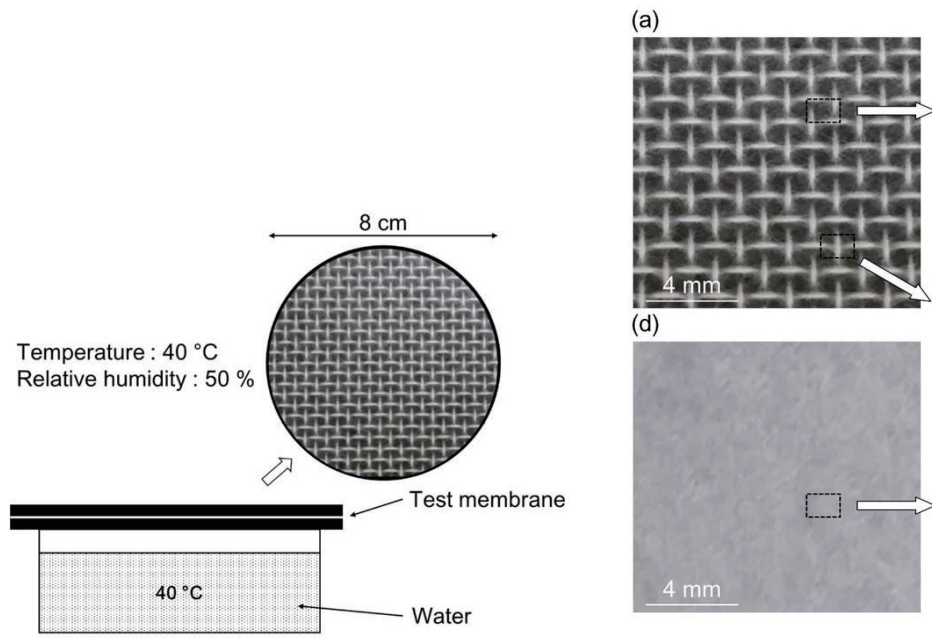

(d)
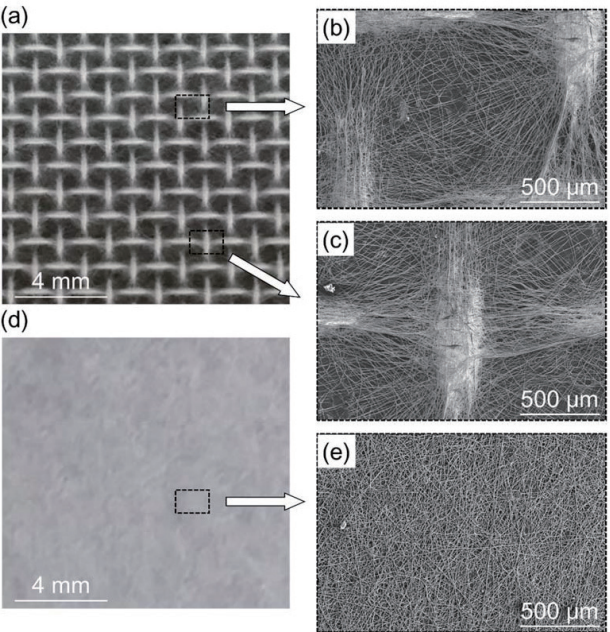

Fig. 3 (left). Schematic of the water-cup method. A circular membrane $8 \mathrm{~cm}$ in diameter covers a cup containing $40{ }^{\circ} \mathrm{C}$ water in an environment of $40{ }^{\circ} \mathrm{C}$ and $50 \%$ relative humidity.

Fig. 4 (right). (a) Optical image of the PU-mesh membrane. (b) and (c) SEM images of the PUmesh membrane focused on a scarcely electrospun area and a thickly electrospun area, respectively. (d) Optical image of the PU-foil membrane. (e) SEM image of the PU-foil membrane focused on a random area. 
mesh of metal wires, and sparse regions corresponding to the gaps in the mesh. Figure 4(b) shows an SEM image of a sparse region, and Fig. 4(c) shows an SEM image of a thick area. Figure 4(d) shows an optical image of the PU-foil membrane. The randomly oriented electrospun PU fibers were consistent in terms of fiber distribution across the membrane. Figure 4(e) shows an SEM image of the PU-foil membrane.

Note that an identical volume of PU solution was used to fabricate both membranes $(0.4 \mathrm{~mL})$. Therefore, the PU fibers in each membrane should be of identical total length, with the only difference being in their location and orientation. In Figs. 4(b) and 4(c), we can see that the distribution of the fibers reflected the geometry of the mesh electrode, whereas the membrane formed using the planar collector electrode resulted in a uniform membrane, as shown in Fig. 4(e). Larger pores are expected in the sparse regions in the PU-mesh membrane and, because of the larger pores, the PU-mesh membrane is expected to exhibit improved breathability.

Figure 5 shows the average pore sizes of the two membranes. The average pore size of the PU-foil membrane was $8.6 \mu \mathrm{m}$, compared with $9.6 \mu \mathrm{m}$ for the PU-mesh membrane. These results suggest that varying the geometry of the electrode enabled control of the membrane morphology and pore size.

\subsection{Membrane thickness}

Figure 6(a) shows an SEM image of the PU-mesh membrane, showing a thick region (i.e., above the wires in the metal mesh). Also shown is a two-dimensional schematic diagram of the cross-sectional area from regions (i) to (ii) in Fig. 6(a) revealing a thicker region in the center.

The membrane was cut along a line that shows the transition between regions (i) and (ii), and was attached to a vertical substrate, with the (i)-(ii) transition facing upward. Figure 6(b) shows an SEM image of the vertical PU-mesh membrane, revealing the crosssectional view, which is shaded in white. The boundary of the shaded area was determined along the trace of the fibers to represent the cross-sectional area. A further

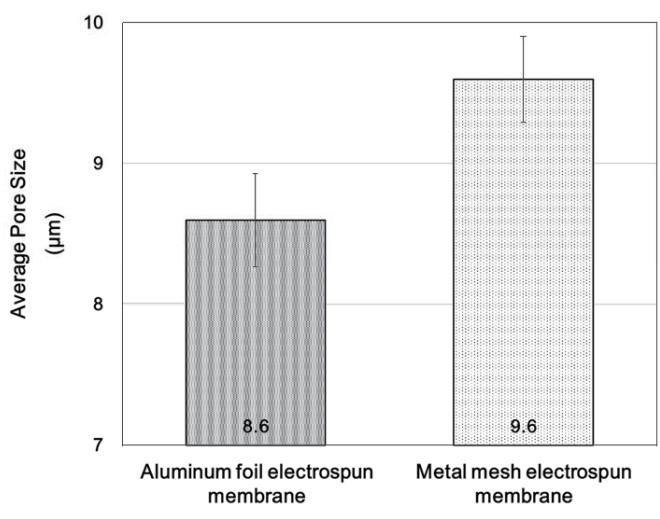

Fig. 5. Comparison of the average pore sizes of the PU-foil and PU-mesh membranes. 

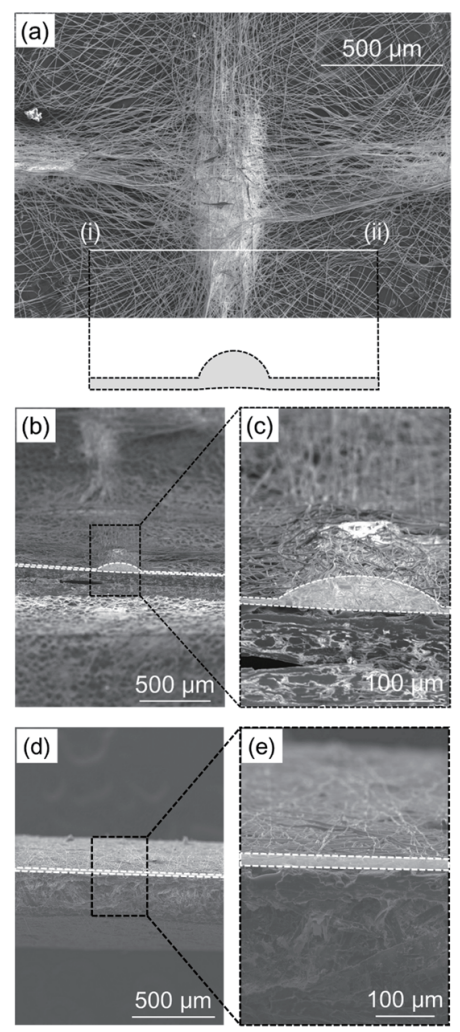

Fig. 6. (a) SEM image of the PU-mesh membrane focused on a thickly electrospun area. Underneath is the schematic diagram showing the cross-sectional area of (i)-(ii). (b) SEM image of the vertically standing PU-mesh membrane with the cross section heading upward. The shaded area represents the cross-sectional area. (c) Further magnified SEM image of the PU-mesh membrane's cross-sectional area. (d) SEM image of the vertically standing PU-foil membrane with the cross section heading upward. (e) Further magnified SEM image of the PU-mesh membrane's cross-sectional area.

magnified image is shown in Fig. 6(c), and Figs. 6(d) and 6(e) show SEM images of the vertical PU-foil membrane. The thickest part of the PU-mesh membrane was approximately $60 \mu \mathrm{m}$ thick, and the thinnest part was approximately $3 \mu \mathrm{m}$ thick, which corresponds to one or two fibers (the individual fibers are $1-2 \mu \mathrm{m}$ in diameter). The PUfoil membrane was approximately $19 \mu \mathrm{m}$ thick throughout the entire area.

According to Darcy's law, the thickness of a membrane is important for determining the flow of gas molecules through that membrane, which is directly related to the breathability. ${ }^{(26)}$ The flow of gas molecules can be expressed as

$$
Q=-\frac{k A}{\mu} \cdot \frac{P_{\mathrm{b}}-P_{\mathrm{a}}}{L},
$$




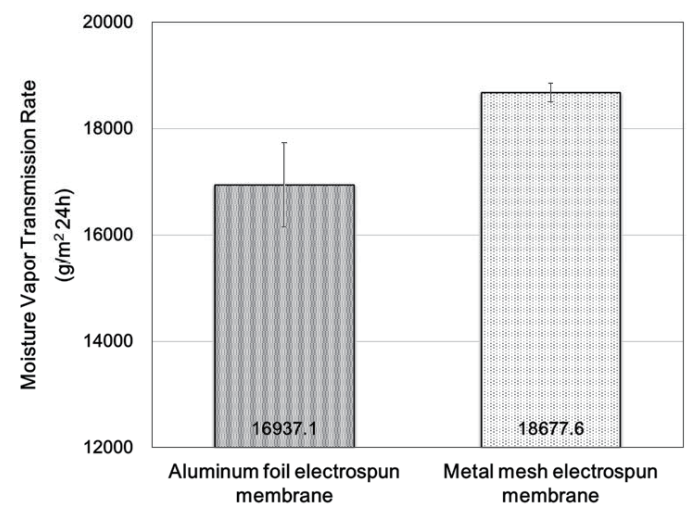

Fig. 7. Comparison of MVTRs of PU-foil and PU-mesh membranes.

where $L$ is the length of the medium through which the gas molecules pass (which in this case is the thickness of the membrane), $k$ is the intrinsic permeability of the medium, $A$ is the cross-sectional area, $\left(P_{\mathrm{b}}-P_{\mathrm{a}}\right)$ is the pressure drop, and $\mu$ is the viscosity of the fluid.

Assuming that the environment remains constant, the relative difference in $Q$ between the two membranes can be calculated from the thickness. For the PU-mesh membrane, which has regions of different thicknesses, the relative area of the two regions was taken into consideration to calculate the average $Q$. The ratio of the two regions was assumed to be identical to that of the mesh used to form the membrane. The calculated $Q$ value of the PU-mesh membrane was 3.35-fold larger than that of the PU-foil membrane.

\subsection{Breathability}

Figure 7 shows the MVTR of the two membranes. The MVTR of the PU-mesh membrane was $18677.6 \mathrm{~g} / \mathrm{m}^{2}$ over $24 \mathrm{~h}$, and that of the PU-foil membrane was 16937.1 $\mathrm{kg} / \mathrm{m}^{2}$ over $24 \mathrm{~h}$. The difference in these figures is clearly smaller than a factor of 3 , which would be expected on the basis of Darcy's law for the permeability of gas molecules. Further modeling work, which takes into account the geometry of the membrane in the calculation of breathability, is therefore required.

\section{Conclusions}

We have described the fabrication of electrospun PU fabrics using metal mesh and planar aluminum foil collectors to investigate the effects of controlling the morphology of the electrospun membrane on its breathability. The PU membranes fabricated using the mesh exhibited larger pore sizes than those fabricated using the planar aluminum foil; thus, higher flow rates of vapor-phase molecules are expected. We fabricated a breathable PU membrane by electrospinning the polymer using a metal mesh collector, and demonstrated enhanced breathability compared with the membrane fabricated using a planar collector electrode. This was explained by considering the structure of the 
breathable PU membrane, which had a larger average pore size, which is expected to lead to a higher flow rate of gas molecules. In a future work, we plan to investigate the dependence of breathability on the dimensions of the metal mesh used to form the fabric, and anticipate further improvements in breathability by optimizing the geometry of the collector electrode.

\section{Acknowledgements}

This work was supported by the National Research Foundation of Korea (NRF) grant funded by the Korean government (MSIP) (No. 2011-0030075), the Industrial Technology Innovation Program (No. 10048358) funded by the Ministry of Trade, Industry and Energy (MI, Korea), and the Basic Science Research Program through the NRF grant funded by the Ministry of Science, ICT \& Future Planning (No. 2014R1A1A1002567).

\section{References}

1 Y. K. Kang, C. H. Park, J. Kim and T. J. Kang: Fibers Polym. 8 (2007) 564.

2 B. Yoon and S. Lee: Fibers Polym. 12 (2011) 57.

3 H. S. Lim, J. H. Baek, K. Park, H. S. Shin, J. Kim and J. H. Cho: Adv. Mater. 22 (2010) 2138.

4 S. Ramakrishna, K. Fujihara, W. Teo and T. Yong: Mater. Today. 9 (2006) 40.

5 D. Li and Y. Xia: Adv. Mater. 16 (2004) 1151.

6 A. Greiner, J. H. Wendorff: Angew. Chem. Int. Ed. 46 (2007) 5670.

7 Y. Dzenis: Mater. Sci. 304 (2004) 1917.

8 A. Gañán-Calvo: Phys. Rev. Lett. 79 (1997) 217.

9 D. H. Reneker, A. L. Yarin, H. Fong and S. Koombhongse: J. Appl. Phys. 87 (2000) 4531.

10 D. H. Reneker and I. Chun: Nanotechnology. 7 (1996) 216.

11 X. Qin and S. Wang: J. Appl. Polym. Sci. 109 (2008) 951.

12 A. Martins, J. V Araújo, R. L. Reis and N. M. Neves: Nanomedicine (Lond) 2 (2007) 929.

13 J.-A. Park, J. Moon, S.-J. Lee, S. H. Kim, T. Zyung, and H. Y. Chu: Mater. Lett. 64 (2010) 255.

14 L. M. Bellan and H. G. Craighead: J. Vac. Sci. Technol. B Microelectron. Nanom. Struct. 24 (2006) 3179.

15 C. Chang, K. Limkrailassiri and L. Lin: Appl. Phys. Lett. 93 (2008) 1.

16 A. Theron, E. Zussman and A. Yarin: Nanotechnology. 12 (2001) 384.

17 E. Zussman, A. Theron and A. L. Yarin: Appl. Phys. Lett. 82 (2003) 973.

18 D. Li, Y. Wang and Y. Xia: Nano Lett. 3 (2003) 1167.

19 D. Li, Y. Wang and Y. Xia: Adv. Mater. 16 (2004) 361.

20 D. Li, G. Ouyang, J. T. McCann and Y. Xia: Nano Lett. 5 (2005) 913.

21 D. Zhang, and J. Chang: Nano Lett. 8 (2008) 3283.

22 B. Kim, S. J. Cho, T. An, H. Ryu, H. and Lim, G. Lim: Phys. Status Solidi RRL 6 (2012) 409.

23 S. J. Cho, B. Kim, T. An and G. Lim: Langmuir 26 (2010) 14395.

24 C. Vaquette and J. J. Cooper-White: Acta Biomater. 7 (2011) 2544.

25 S. J. Cho, H. Nam, H. Ryu and G. Lim: Adv. Funct. Mater. 25 (2013) 5577.

26 S. Whitaker and Transp: Porous Media 1 (1986) 3. 